\title{
Statistics of Decision Making in the Leech
}

\author{
Elizabeth Garcia-Perez, ${ }^{1 \star}$ Alberto Mazzoni, ${ }^{1 \star}$ Davide Zoccolan, ${ }^{1 \star}$ Hugh P. C. Robinson, ${ }^{2}$ and Vincent Torre ${ }^{1}$ \\ ${ }^{1}$ Scuola Internazionale Superiore di Studi Avanzati, 34014 Trieste, Italy, and ${ }^{2}$ Physiological Laboratory, University of Cambridge, Cambridge CB2 3EG, \\ United Kingdom
}

\begin{abstract}
Animals continuously decide among different behaviors, but, even in invertebrates, the mechanisms underlying choice and decision are unknown. In this article, leech spontaneous behavior was tracked and quantified for up to $12 \mathrm{~h}$. We obtained a statistical characterization, in space and time domains, of the decision processes underlying selection of behavior in the leech. We found that the spatial distribution of leech position in a uniform environment is isotropic (the same in all directions), but this isotropy is broken in the presence of localized external stimuli. In the time domain, transitions among behaviors can be described by a Markov process, the structure of which (allowed states and transitions) is highly conserved across individuals. Finally, a wide range of recurrent, deterministic motifs was identified in the apparently irregular and unstructured exploratory behavior. These results provide a rigorous description of the inner dynamics that control the spontaneous and continuous flow of behavioral decisions in the leech.
\end{abstract}

Key words: leech behavior; dynamics; recurrence plot; periodic motion; exploratory motion; Markov process

\section{Introduction}

Animals react to changes of the environment by responding with an appropriate behavior (Tinbergen, 1951; Drewes, 1984), from simple escape responses in invertebrates (Furshpan and Potter, 1959; Zucker, 1972; Bennett, 1997, 2000) to decision strategies in primates (Barraclough et al., 2004). An understanding of the decision process and the neuronal mechanisms underlying the selection of a specific behavior is a fascinating problem of contemporary neuroscience (Glimcher, 2002; Montague and Berns, 2002). As the pioneering work of sensory psychology and psychophysics (Green and Swets, 1966), decision making has been investigated primarily by looking at the behavioral responses of human subjects. More recently, neural correlates underlying decision processes have been investigated in primates performing visual recognition tasks (Freedman et al., 2001; Gold and Shadlen, 2002; Barraclough et al., 2004) and by using microstimulation of cortex areas involved in the representation of sensory stimuli (Salzman et al., 1990; Britten and Van Wezel, 1998; Ditterich et al., 2003). Similar experimental paradigms in simple invertebrates, such as the leech, revealed the existence of nontrivial interactions between different sensory modalities and trigger-like command neurons (Brodfuehrer and Friesen, 1986a,b,c; Brodfuehrer and Burns, 1995; Esch et al., 2002).

Approaches based on detection/decision tasks provide a solid framework for investigating the decision process in response to external sensory stimuli but are less suitable for understanding

Received Sept. 14, 2004; revised Jan. 16, 2005; accepted Jan. 18, 2005.

We thank Dr. William Kristan for valuable scientific suggestions and helpful comments on this manuscript.

*E.G.-P., A.M., and D.Z. contributed equally to this work.

Correspondence should be addressed to Vincent Torre, Scuola Internazionale Superiore de Studi Avanzati, Via Beirut 2, 34014 Trieste, Italy. E-mail: torre@sissa.it.

D. Zoccolan's present address: McGovern Institute for Brain Research, Massachusetts Institute of Technology, Cambridge, MA 02139.

DOI:10.1523/JNEUROSCI.3808-04.2005

Copyright $\odot 2005$ Society for Neuroscience $\quad$ 0270-6474/05/252597-12\$15.00/0 the inner neural dynamics that control spontaneous transitions from one behavioral category to another when animals are in an almost uniform and stable environment. This issue needs an alternative approach based on the analysis of the temporal structure of unconstrained animal behavior, which has been applied successfully in the analysis of behavior of different species (Bressers et al., 1995; Haccou and Meelis, 1992; Chen et al., 2002); however, this requires a reliable classification of all behavioral categories and continuous measurement of all possible transitions between these categories over a period of many hours.

In our work, we tried to overcome such difficulties by quantifying the behavior of the medicinal leech, the sensory capabilities and neuronal circuitry of which are relatively well understood (Baader and Kristan, 1992; Brodfuehrer and Thorogood, 2001; Esch et al., 2002). The animal was positioned in an almost homogeneous, stable, and isotropic environment: a large dish filled with dechlorinated water at a constant temperature and illuminated by dim, diffused light. An automatic system based on imaging with a CCD camera tracked three colored beads attached to the back of the leech and recorded its motion for up to $12 \mathrm{~h}$. Statistical analysis based on the kinematic and spectral content of the leech motion (Mazzoni et al., 2005) allowed unambiguous classification of $>90 \%$ of the leech motion into characteristic behavioral categories. We found that the mechanism by which the leech decides its behavior is adequately described by a Markov process, the transition rates of which are probably controlled by the firing of command-like neurons found in the leech head ganglion (Brodfuehrer and Burns, 1995; O'Gara and Friesen, 1995; Shaw and Kristan, 1997; Esch et al., 2002).

\section{Materials and Methods}

Animals and preparations

Adult leeches (Hirudo medicinalis) were obtained from Ricarimpex (Eysines, France) and kept at $5^{\circ} \mathrm{C}$ in tap water dechlorinated by aeration for $24 \mathrm{~h}$. Two different kinds of preparations were used. The first preparation consisted of an intact leech free to move on a dish. The second prepara- 
Table 1. Summary of the behaviors of the leech and criteria used for their identification

\begin{tabular}{|c|c|c|}
\hline Behaviors & Description & Behavior identification \\
\hline Stationary & Anterior and posterior sucker attached to the dish & Speed of the head $V_{\text {head }}(n)$ and $V_{\text {tail }}(n)<1$ pixel $/ \mathrm{s}$ \\
\hline Still & Midbody still & Speed of the midbody $V_{\text {midbody }}(n)<1 \mathrm{pixel} / \mathrm{s}$ \\
\hline Peristaltic-like & Midbody oscillating in a regular way & $V_{\text {midbody }}(n)>1$ pixel $/ \mathrm{s}$, oscillating with a frequency of $\sim 0.03 \mathrm{~Hz}$ \\
\hline Head attached & Midbody oscillating in an irregular way & $V_{\text {midbody }}(n)>1 \mathrm{pixel} / \mathrm{s}$ with no regular oscillations \\
\hline Swimming & Undulatory movement of the entire body & $\begin{array}{l}\text { Maximal speed of the head } V_{T}^{\max }(n) \text { between } 10 \text { and } 30 \text { pixels } / \mathrm{s} \text {; } \\
\text { dominant frequency } f_{T}^{d}(n) \text { of elongation (head to tail distance) } \\
\text { between } 1.3 \text { and } 1.7 \mathrm{~Hz} \text {; and speed of the tail } V_{T}^{\text {tail }}(n)>3 \text { pixels/s }\end{array}$ \\
\hline Pseudo-swimming (ventilation) & $\begin{array}{l}\text { Undulatory movement of the body and posterior sucker } \\
\text { attached to the dish }\end{array}$ & $\begin{array}{l}V_{T}^{\max }(n) \text { between } 10 \text { and } 30 \text { pixels } / s ; f_{T}^{d}(n) \text { of elongation between } \\
1.3 \text { and } 1.7 \mathrm{~Hz} \text {; and } V_{T}^{\text {tail }}(n)<3 \text { pixels } / \mathrm{s}\end{array}$ \\
\hline Crawling & $\begin{array}{l}\text { Alternating steps of elongation (posterior sucker attached) } \\
\text { and contraction (anterior sucker attached) }\end{array}$ & $\begin{array}{l}V_{T}^{\max }(n) \text { between } 20 \text { and } 40 \text { pixels } / s ; f_{T}^{d}(n) \text { of elongation between } \\
0.16 \text { and } 0.24 \mathrm{~Hz}\end{array}$ \\
\hline Exploratory & $\begin{array}{l}\text { Irregular oscillations of the head and anterior part of the } \\
\text { body with the posterior sucker attached }\end{array}$ & $V_{T}^{\max }(n)$ between 10 and 50 pixels $/ s ; f_{T}^{d}(n)$ of elongation $<0.12 \mathrm{~Hz}$ \\
\hline Abrupt movements & Rapid transitions between two states & Changes in position of at least 20 pixels in $<5 \mathrm{~s}$ \\
\hline
\end{tabular}

tion consisted of an intact leech restrained by fixing the rear sucker of the animal to the center of the dish with one pin. Pins were carefully inserted through the animal's body, avoiding the penetration of the connective fibers running along the animal. When one pin was used, the leech was able to fully rotate around the pin and could explore the surrounding space.

All preparations were kept either in a round dish (15 cm diameter) or a square tank $(15 \times 15 \mathrm{~cm})$ filled with dechlorinated water at constant temperature $\left(20-24^{\circ} \mathrm{C}\right)$ and illuminated by dim, diffused light, with no abrupt spatial and/or temporal gradients of sensory stimuli, such as chemical or olfactory anisotropies, isolated visual cues, or abrupt illumination changes. The dechlorinated water in the dish was changed every $2 \mathrm{~h}$ to avoid the effects of serotonin and ions released by the leech (Schnizler and Clauss, 1998; Schnizler et al., 2002). In every preparation, three colored plastic beads (red, green, and blue) of a diameter of $\sim 2 \mathrm{~mm}$ were glued on the back of the leech with Superglue (World Precision Instruments, Berlin, Germany). The red, green, and blue beads were attached near the head, the middle, and the tail of the leech.

\section{Imaging}

A color CCD camera $(640 \times 480$ pixels of image size; model $231 S$; Watec, Tsuruoka, Japan) was used to view the leech from above. The camera was connected via the S-video-output to a frame grabber (PCI-1411; National Instruments, Milan, Italy) installed on a personal computer, which processed the images in real time. The colored beads placed on the back of the leech were tracked at $10 \mathrm{~Hz}$ by using a software program developed by Sergio Graziosi in our laboratory (Mazzoni et al., 2005) with LabVIEW 6.1 (National Instruments). Briefly, the software acquires images directly in the hue/saturation/lightness color space, and the tracking algorithm works in real time with no frequency constraints. A usercustomized color matching is performed to convert each acquired frame into three binary images, one for each bead, in which the ON bit represents a pixel that fit in the expected color subspace. This software did not require saving all of the processed images but only the coordinates of the selected beads. In this way, it was possible to monitor the leech behavior for several hours without filling hard disk space. The positions of the beads on the image plane were acquired as Cartesian coordinates, and six time series were obtained as follows:

$$
\begin{aligned}
& {[x(n), y(n)]_{\text {head/red; }}} \\
& {[x(n), y(n)]_{\text {midbody/green }} ;} \\
& {[x(n), y(n)]_{\text {tail/blue }} \text { for } n=1 \ldots N \text { steps }}
\end{aligned}
$$

where every step corresponds to $100 \mathrm{~ms}$.

Some analysis required the motion to be studied in a polar system centered on the middle of the dish. In this way, Cartesian coordinates $(x, y)_{\text {color }}$ were transformed into a pair of planar polar coordinates $(\rho, \theta)_{\text {color }}$. When the leech was restrained from moving around by a pin inserted in its tail, the polar system had a clear geometrical and physiological meaning: $\rho_{\text {color }}$ is the elongation of the leech body at the position marked by the bead (color), and $\theta_{\text {color }}$ is the angle between this position and a reference axis.

\section{Sensory stimulation}

In one series of experiments, a small pulsating light-emitting diode (LED) (at $0.5 \mathrm{~Hz}$ ) was put above the water but near the edge of the dish. The behavior of the leech was first monitored for $3 \mathrm{~h}$ in control conditions of low and diffused light (without light stimulation). The animal was allowed to rest for $30 \mathrm{~min}$, and then the light stimulation (LED) was initiated. In some experiments, two LEDs were placed diametrically opposite each other and flashed alternately at $1 \mathrm{~s}$ intervals. These experiments were done with restrained leeches, and the residence time distributions, in both light and control (no light) preparations, were analyzed.

\section{Behavior classification}

Often the leech moved in the dish for $>12 \mathrm{~h}$. The marker coordinate time series thus often comprised $>100,000$ samples, which had to be analyzed consistently. We therefore developed an automatic classification method for identifying the leech behavior, which is based on analyzing the kinematics and spectral content of the recorded time series and is described in detail in a previous work (Mazzoni et al., 2005).

Briefly, leech movements were classified into six different categories (Table 1): stationary (including still, peristaltic-like, and head-attached states), swimming, pseudo-swimming (or ventilation states), crawling, and exploratory and abrupt movements. At each time step $n$ of the recorded time series, different criteria were used to discriminate one behavioral category from the others. For example, given the set of coordinates describing the leech dynamics at time step $n$, to test whether the current behavioral state was a stationary state, the speed of the head $\left[V_{\text {head }}(n)\right]$ and tail $\left[V_{\text {tail }}(n)\right]$ beads at time $n$ was calculated. If the speed was less than 1 pixel per second (pixel/s), then the state was considered stationary. Additionally, to distinguish between the three different stationary states, the speed of the midbody $\left[V_{\text {midbody }}(n)\right]$ bead and its frequency were computed. If $V_{\text {midbody }}(n)$ was $<1 \mathrm{pixel} / \mathrm{s}$, then the state was classified as still; however, if $V_{\text {midbody }}(n)$ was $>1$ pixel/s, then the frequency of oscillation was computed, and, depending on this result, the stationary state was classified as peristaltic like (with a frequency of $\sim 0.03$ $\mathrm{Hz}$ ) or head attached, during which the midbody oscillated in an irregular way.

The other behavioral categories were identified by computing the following parameters: (1) elongation $e(n)$, that is, the distance from head to tail beads; (2) maximal speed $V_{T}^{\max }(n)$ of the head over a time window of width $T$ centered in $n$ (with $T$ ranging from 20 to $50 \mathrm{~s}$ ); (3) dominant frequencies $f_{T}^{l}(n)$ of the power spectrum $\operatorname{PS}_{n}^{T}(f)$ of $e(n)$, computed over the same time window of width $T$. More specifically, oscillatory behaviors (such as swimming, pseudo-swimming, and crawling) could be reliably identified by the pairs of values $\left[V_{T}^{\max }(n), f_{T}^{d}(n)\right]$. For instance, swimming episodes were consistently characterized by $f_{20}^{d}(n) \sim 1.5 \mathrm{~Hz}$ and $V_{20}^{\max }(n) \sim 20$ pixels/s, whereas crawling was characterized by $f_{50}^{d}(n)$ 
$\sim 0.2 \mathrm{~Hz}$ and $V_{50}^{\max }(n) \sim 30$ pixels/s. The spectral analysis of $e(n)$ also allowed identification of the exploratory states, because events with $f_{50}^{d}(n)$ at the lowest resolved frequencies $(\sim 0.1 \mathrm{~Hz})$ matched correctly with the exploratory movements (i.e., slow and irregular oscillations of the head and the anterior part of the body). An exhaustive inventory of all identified leech behaviors and the criteria used to classify them is given in Table 1. Note that all definitions of behavior are based on units of pixels, in which 1 pixel $=0.4 \mathrm{~mm}$.

Statistical analysis of stationary, swimming, and exploratory states Space distribution. Once a specific behavior was identified across the whole time series, the mean values of the polar coordinates for the head $\left[\rho_{\text {behav }}(i), \theta_{\text {behav }}(i)\right]$ in each time interval $i=1, \ldots, N_{\text {behav }}$ in which the leech was performing that behavior were computed $\left(N_{\text {behav }}\right.$ is the total number of episodes found for that behavior). The distributions of angles $\theta_{\text {behav }}(i)$ were plotted as polar histograms (see Fig. $2 B, D$ ), with an angular bin size of $18^{\circ}$. If the analyzed behavior is isotropic, then the number of recorded states with $\theta_{\text {behav }}(i)$ belonging to a given angular sector must be distributed according to a Poisson distribution with mean $\mu=$ (number of occurrences $N_{\text {behav }}$ )/(number of sectors $S$ ). A $\chi^{2}$ test was used to check whether the obtained distributions were Poisson distributions $(p>0.05)$ and therefore whether the behavior was isotropic. If the behavior was found to be significantly anisotropic, then a post hoc test was used to specifically test whether such anisotropy was caused by one or more sectors that were overpopulated and to find out the orientation of such sectors (i.e., the preferred direction for that behavior). The post hoc test was based on the null hypothesis that the distribution of states per sector is not significantly different from a Poisson distribution and that therefore the probability of having $Z$ states or less in a sector is the following:

$$
P_{\mu}(n \leq Z)=\sum_{i=0}^{Z} P_{\mu}(i) .
$$

Then, the probability $Q$ that $n$ exceeds $Z$, at least in one sector, is the following:

$$
Q(Z)=1-\left(\sum_{i=0}^{Z} P_{\mu}(i)\right)^{S}
$$

In Figure $2 B-D$, the magenta circle indicates the value of $Z$ associated with $Q=0.05$. Note that this value depends only on the number of states and the number of sectors.

Time series correlation. To study the linear properties of the sequence of identified states, series of average angles $\theta_{\text {behav }}(i)$ and lengths $\rho_{\text {behav }}(i)$, with $i=1, \ldots, N_{\text {behav }}$, were considered as the outcome of two stochastic processes $\Theta_{\text {behav }}(i)$ and $\mathrm{P}_{\text {behav }}(i)$. First, we assessed whether these stochastic processes were stationary, testing the stability of the mean and variance on the first and second half of the series (ANOVA test).

The correlation between consecutive values $\theta_{\text {behav }}(i)$ [and consecutive values $\rho_{\text {behav }}(i)$ ] was then evaluated by computing the autocovariance of the process $\Theta_{\text {behav }}(i)$ [and of the process $\mathrm{P}_{\text {behav }}(i)$ ]. Similarly, the correlation between consecutive pairs of values $\theta_{\text {behav }}(i)$ and $\rho_{\text {behav }}(i)$ was evaluated by computing the covariance between the processes $\Theta_{\text {behav }}(i)$ and $\mathrm{P}_{\text {behav }}(i)$. Statistical significances of autocorrelation and crosscorrelation values were evaluated by a two-tailed normal test under the assumption that correlation values for uncorrelated time series are normally distributed with mean zero and variance $1 /(N-l-3)$, where $N$ is the number of samples in the two series, and $l$ is the lag number. Correlation values were considered significant at $p<0.05$. The number of consecutive significantly correlated states around $n=0$ gives the size of the central peak of the autocorrelation. The width of the central peak of the autocovariance function gives a measure of the "memory" of the stochastic process and indicates on how many previous states the identity of the state depends (Priestley, 1981). To compare the processes $\Theta_{\text {behav }}(i)$ and $\mathrm{P}_{\text {behav }}(i)$ with random processes of the same statistical properties but without any correlation ("no memory"), the original series $\rho_{\text {behav }}(i)$ and $\theta_{\text {behav }}(i)$ were scrambled before the autocovariance was computed.
Time structure of the sequence of behaviors. The time structure of the sequence of behavioral states was studied by computing, for any identified behavior, the distribution of the duration $\Delta t$ of the time intervals (residence times) during which that behavior was performed. In every experiment and for every behavior, this distribution had a high and narrow peak centered on a small $\Delta t$ (usually $<10 \mathrm{~s}$ ), which decayed to 0 with increasing $\Delta t$. This suggested that the sequence of behaviors could be modeled as a Markov process in which the residence time for each behavior is distributed as an exponential or a mixture of exponentials (Haccou and Meelis, 1992). Therefore, for each behavior, the observed residence time distribution was fit by an exponential function, $f(\Delta t)=$ $A \exp (-\Delta t / \tau)$, or by the sum of two exponential functions. To determine whether two exponentials were required, goodness of fit was tested with a $\chi^{2}$ test, and a one-exponential fit was accepted only if there was no significant difference $(p>0.05)$ between the data and the fit. When one exponential was insufficient, two exponentials were always found to be sufficient by the $\chi^{2}$ criterion.

Power spectral analysis. An informative parameter about the motion of the leech is the elongation $e(n)$, i.e., the distance between the head and the tail, which varies during every oscillatory behavior. As mentioned previously, during the classification procedure (Mazzoni et al., 2005), for every nonstationary point in the time series, a power spectrum $\operatorname{PS}(f)$ of the elongation over the portion of the time series centered on that point and having a half-size of $10 \mathrm{~s}$ was computed to accurately detect highfrequency oscillations. A second power spectrum with a half-size of $25 \mathrm{~s}$ was then computed to discriminate low-frequency oscillations. Sometimes, power spectra were computed even for much larger windows to analyze global properties of the behavior (see Fig. $4 A, B$ ). Welch's averaged modified periodogram method was used ( $p$ welch function in Matlab; MathWorks, Natick, MA), with $50 \%$ overlap between successive time windows, each one being one-fourth the size of the analyzed interval. Points of the resulting power spectra were averaged within logarithmically spaced frequency bins to smooth the power spectrum at high frequencies. Power spectra were further smoothed by averaging every spectral point PS $(f)$ over a semi-octave centered on each frequency $f$ to enhance detection of any peaks (Mazzoni et al., 2005).

Recurrence analysis of exploratory states. Complex exploratory motion was investigated using the recurrence plot technique (Eckmann et al., 1987). The advantage of this approach is its capability of detecting deterministic or repeated component patterns even in highly nonlinear and irregular time series, which would be lost through averaging in linear measures such as the power spectrum (or equivalently, the autocorrelation function). First, an $m$-dimensional delay representation (Kantz and Schreiber, 1997) is constructed for a $1000 \mathrm{~s}$ segment of motion time series. The delay coordinate vectors $\mathbf{d}_{n}$ are given by the following:

$$
\mathbf{d}_{n}=\left(y_{n-(m-1) v}, y_{n-(m-2) v}, \ldots, y_{n-v}, y_{n}\right)
$$

These represent successive short pieces of the waveform history preceding each point $y_{n}$ in the time series. We used $m=20$ and lag $v=2$. A $4000 \times 4000$ diagonally symmetric matrix $\mathbf{c}$ is then plotted in which the point at row $i$, column $j$ is color coded (in gray scale) according to the Euclidean distance between $\mathbf{d}_{i}$ and $\mathbf{d}_{j}$ as follows:

$$
c_{i j}=\sqrt{\left(d_{i 1}-d_{j 1}\right)^{2}+\left(d_{i 2}-d_{j 2}\right)^{2}+\cdots+\left(d_{i m}-d_{j m}\right)^{2}} .
$$

Within this plot (see Fig. 5), dark colors represent similarity, or low distance, between delay vectors. A sequence of intensely dark points along a line of slope -1 represents a flow in which history repeats itself. We visually scanned the recurrence matrix for diagonal lines satisfying a threshold similarity condition of length at least twice the duration of the delay vector $(>19 \mathrm{~s})$. Having identified one diagonal, all similar repeats within the time series are easily extracted as those diagonal lines that intersect a horizontal (or vertical) line drawn through the center of the first line. The repetition frequency of the pattern is quantified by the number of such sister diagonals (for a diagram of this process, see Fig. 5). Repeats that follow the same trajectory but with a different timescale appear as diagonal lines with different slopes. 
Estimation of transition probabilities and test of sequential dependences. Once every point of the time series has been classified, the behavior of the leech can be seen as a sequence of identified behavioral states. To estimate the transition probabilities between pairs and triplets of behaviors, we computed, for each behavior $A$, the following quantities: the number of occurrences $N_{A}$ of behavior $A$; the number of times $N_{A B}$ that behavior $A$ was followed by behavior $B$ (i.e., the number of transitions $A \rightarrow B$ ), for all possible following behaviors $B$; the number of times $N_{X A}$ that behavior $A$ was preceded by behavior $X$ (i.e., the number of transitions $X \rightarrow$ $A$ ), for all possible preceding behaviors $X$; the number $N_{X A B}$ of transitions $X \rightarrow A \rightarrow B$, for all possible preceding behaviors $X$ and following behaviors $B$. Then, the probabilities of firstorder transitions $A \rightarrow B$ were estimated as $P(B /$ A) $=N_{A B} / N_{A}$. Similarly, the probabilities of second-order transitions $X \rightarrow A \rightarrow B$ were estimated as $P(B / A, X)=N_{X A B} / N_{X A}$. Values of these transition probabilities during the first and second half of the experiments were compared with a $\chi^{2}$ test to check whether the process was stationary. Differences between the two sets were considered significant at $p<0.05$.

To test whether the sequence of behavioral states could be modeled as a first-order Markov chain, we used the $\chi^{2}$ test for first- against second-order dependences in the sequences of identified states (Haccou and Meelis, 1992). Given the null hypothesis that $P(B / A)=P(B /$ $A, X)$, for all triplets $X \rightarrow A \rightarrow B$, we first computed the $\chi^{2}$ test statistics for each behavior $A$ : $\chi_{A}^{2}=\Sigma_{X} \Sigma_{B}\left[N_{X A B}-N_{X A} P(B / A)\right]^{2} / N_{X A} P(B / A)$, where the summation is taken over all behaviors $X$ and $B$, preceding and following $A$. Then, a global $\chi^{2}$ test statistics was computed as $\chi^{2}=$ $\Sigma_{\mathrm{A}} \chi_{A}^{2}$, i.e., taking into account all possible intermediate behaviors $A$. The degrees of freedom of the global $\chi^{2}$ test statistics are $k=b(b-2)^{2}$, where $b$ is the number of different behaviors identified in the analyzed experiments; however, if some of the transitions toward or from $A$ occur rarely or not at all (i.e., with almost zero probability), this number is overestimated. In this case, the correct number of degrees of freedom becomes $k=\Sigma_{A}\left(b-v_{A}-1\right)\left(b-w_{A}-1\right)$, where $v_{A}$ and $w_{A}$ are the number of transitions toward $A$ and from $A$, respectively, that cannot occur (Haccou and Meelis, 1992). Deviations from the null hypothesis of a first-orderdependent Markov chain were considered significant at $p<0.05$.

\section{Results}

Leech behavior was studied by observing leech motion in a dish with a color CCD camera. Leeches that were completely free to move exhibited their usual behavior, i.e., they crawled, swam, explored, and stayed stationary. Leeches restrained by having one pin inserted in the tail moved and explored the dish for several hours before showing any sign of weakness such as, for instance, small responses to touch, low erection of the annulus, or total immobility. The behavior of the leech was monitored, often for up to $12 \mathrm{~h}$, by imaging and tracking three colored beads attached to its back. In total, 25 freely moving leeches and 27 restrained leeches were

B

$F$
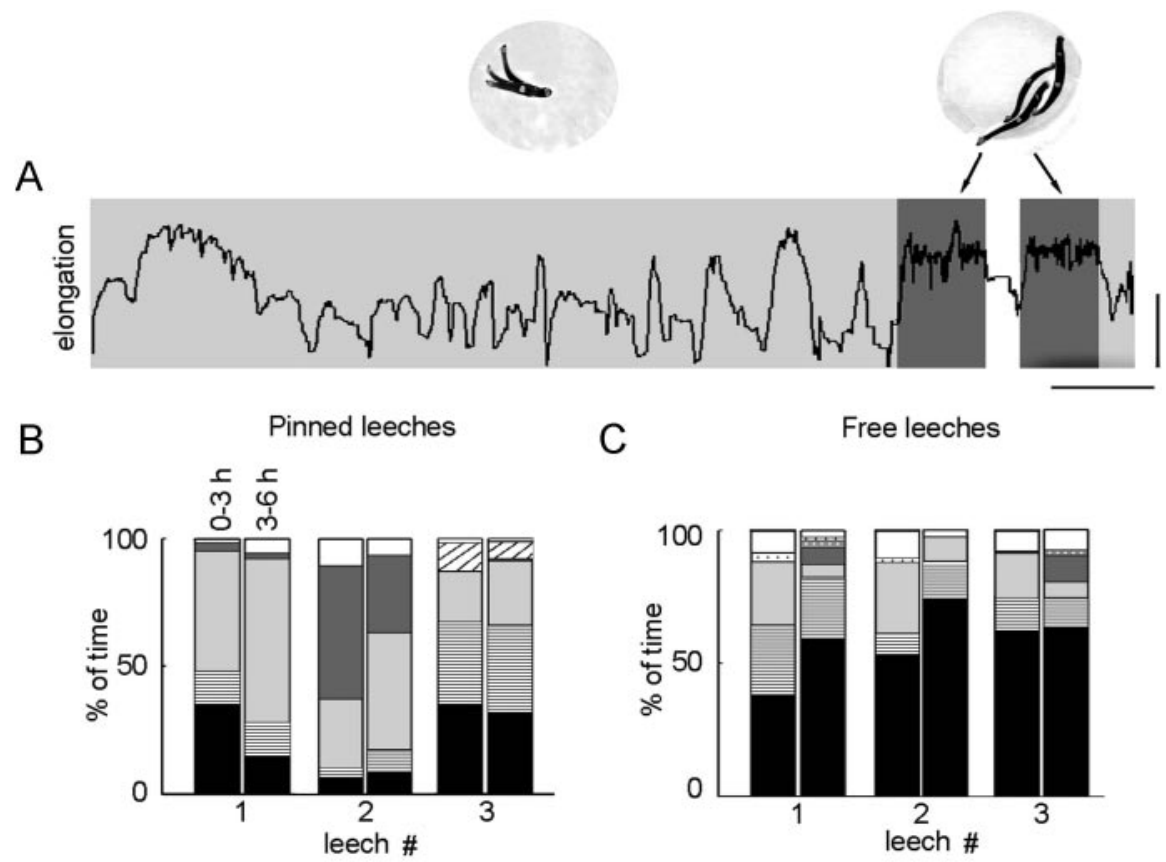

C

Free leeches
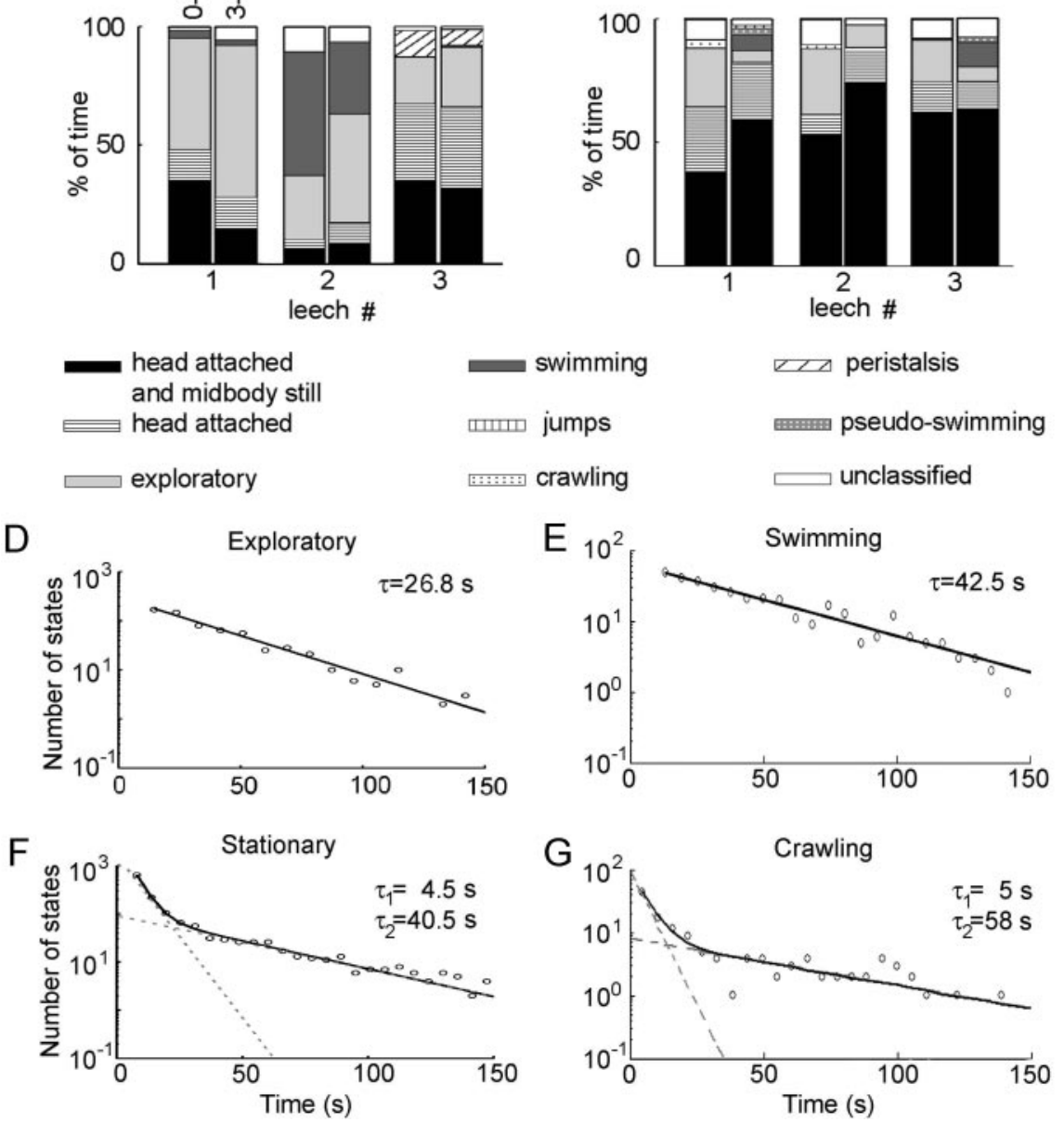

Fure 1. Behaviors of the leech. $A$, Elongation $(n)$ of a freely moving leech tracked over time. Exploratory behaviors are shown in light gray, and swimming behaviors are shown in dark gray. Photos show three superimposed pictures of the leech exploring (left) and three superimposed pictures of the leech swimming (right). $S$ Sale bars, $3.5 \mathrm{~cm} . \boldsymbol{B}$, Time distribution of behaviors for three pinned leeches for the first $3 \mathrm{~h}$ of recording and for the following $3 \mathrm{~h}$. Behaviors are coded as shown at the bottom of $\boldsymbol{B}$. C, As in $\boldsymbol{B}$, but for three free leeches. $\boldsymbol{D}-\boldsymbol{G}$, Distribution of residence time for exploratory ( $\boldsymbol{D}$; pooled data obtained from 7 leeches), swimming ( $\boldsymbol{E} ; 4$ leeches), stationary $(\boldsymbol{F} ; 11$ leeches), and crawling ( $\boldsymbol{G} ; 4$ leeches) states. Duration for each distribution is shown in a semilog plot. Continuous lines represent single- and double-exponential fits for distributions; time constants are indicated.

observed, and their behavior was quantified. Because of the high variability of behaviors from leech to leech (Fig. $1 B, C$ ), only some of these observations were suitable for each particular kind of analysis. For instance, the properties of swimming were quantified from experiments during which the leech was swimming most of the time. As a consequence, the total number of experiments (i.e., leeches) included in each behavioral analysis depends on that particular behavior and analysis. This number is reported in the text and in the legend of each figure. 


\section{Behavior inventory and distribution of the durations of each state}

Figure $1 A$ illustrates the time evolution of the elongation $e(n)$, i.e., distance between the head and tail bead, while the leech moved freely in a covered dish. The analysis of $e(n)$ (Mazzoni et al., 2005) allowed us to identify well known leech behaviors such as crawling and swimming, which are shown in Figure $1 A$ (example of swimming, dark gray, and three superimposed snapshots, right).

By analyzing the motion of the head, midbody, and tail, some other behavioral categories were identified (Mazzoni et al., 2005) and are summarized in Materials and Methods and Table 1. Additionally, there were some unclassified states, characterized by slow drifts or by oscillations superimposed on an apparently exploratory event. One example of this is shown between two swimming states in Figure $1 \mathrm{~A}$ (white). Unclassified states comprised, on average, $\sim 10 \%$ of the observation time.

Leeches not only show stationary and periodic states, but they also exhibit a more complex behavior, referred to here as exploratory (Fig. $1 A$, light gray). This behavioral category is characterized primarily by the absence of any evident regularity (Table 1). Three superimposed snapshots of exploratory behavior are shown in the left inset of Figure $1 A$. Figure $1 B$ shows the relative occurrence of different behavioral categories observed over $6 \mathrm{~h}$ of continuous recording of leech motion (three restrained leeches). For each leech, statistics were obtained from 0 to $3 \mathrm{~h}$ (left bar) and from 3 to $6 \mathrm{~h}$ (right bar), respectively. Figure $1 C$ shows the same statistics but for three freely moving leeches. These data indicate a degree of variability among individuals, but the repertoire of behaviors identified for a given animal as well as the percentage of time spent in each behavior were well preserved during the first and second $3 \mathrm{~h}$ of observation.

The distribution of the residence times of exploratory, swimming, stationary, and crawling states is shown in Figure $1 D-G$. The distributions for exploratory and swimming states could be reliably fitted ( $\chi^{2}$ test; $p>0.05$ ) by a single-exponential function with time constants $\tau=26.8 \mathrm{~s}$ and $\tau=42.5 \mathrm{~s}$, respectively (Fig. $1 D, E)$. On the other hand, the distributions of the residence time for stationary and crawling states were significantly different $\left(\chi^{2}\right.$ test; $p<0.05$ ) from the best fit achieved with a single-exponential function; however, in both cases, the sum of two exponential functions gave a best fit that did not significantly differ from the observed distribution ( $\chi^{2}$ test; $\left.p>0.05\right)$. The time constants found were $\tau_{1}=4.5 \mathrm{~s}$ and $\tau_{2}=40.5 \mathrm{~s}$ for stationary states and $\tau_{1}$ $=5 \mathrm{~s}$ and $\tau_{2}=58 \mathrm{~s}$ for crawling states (Fig. $1 F, G$ ). All of these fits have been calculated on distributions of pooled states from many leeches ( 7 for exploratory, 4 for swimming, 11 for stationary, and 4 for crawling) because single experiments did not have enough data to provide a reliable evaluation of the time constants; however, after the best fit for each pooled distribution was found, we tested whether individual distributions were consistent with that fit. The results of this second test are as follows: six of seven residence time distributions of exploratory states, four of four distributions of swimming states, 11 of 11 of stationary states, and four of four distributions of crawling states were not significantly different $\left(\chi^{2}\right.$ test; $\left.p>0.05\right)$ from the corresponding fitting functions obtained with the pooled data.

\section{Spatial distribution}

Leeches appear to wander around uniformly. Indeed, when the tail is pinned to the center of a round or square dish, the leech explores uniformly in every direction (Fig. $2 A$ ), and the orientation of its body during stationary states is evenly distributed over all $\theta$ coordinates. To test the isotropy of leech orientation during stationary states, the area of the dishes was divided into 20 sectors of $18^{\circ}$ each (Fig. 2 B). To have expected frequencies of at least five states per sector, seven experiments were done with pinned leeches, with at least 100 stationary states each, were analyzed. In seven of seven leeches, the distribution of the number of states per sector was not significantly different from a Poisson distribution ( $\chi^{2}$ test; $\left.p>0.05\right)$, as expected for an isotropic behavior. As an example, four superimposed representative polar plot distributions (a different color for each leech) are shown in Figure $2 \mathrm{~B}$. These distributions show the leech orientation for the first 100 stationary states. The magenta circle indicates the confidence threshold for each sector, with $p<0.05$ of being exceeded (see Materials and Methods), given the null hypothesis of isotropic behavior. The radius of this circle depends only on the number of states analyzed and on the number of sectors and therefore is the same for every data set analyzed in the figure. If one sector exceeds this confidence threshold, then the distribution of states is anisotropic, because the animal visited that sector a significantly higher number of times than the others. For the experiments shown in Figure 2, because no sector exceeded this threshold, there was no significant deviation from isotropy. Similar distributions were obtained for all experiments $(n=7)$.

Free leeches spent most of the time near the dish edges (Fig. $2 C$ ), but again the $\theta$ distribution of stationary states did not have any statistically privileged direction. For this analysis, five experiments with free leeches with at least 80 stationary states were analyzed (just three of them having $>100$ states). The number of occurrences per sector followed a Poisson distribution in five of five leeches $(p>0.05)$. As an example, the superimposed polar distributions for the first 50 stationary states observed in four different leeches (to simplify the plot) are shown in Figure $2 D$ with their confidence threshold.

\section{Temporal distribution}

Elongations $\rho$ and orientations $\theta$ during stationary states were ordered according to their temporal occurrence, and their temporal statistics were evaluated. Autocorrelation analysis for both $\rho$ and $\theta$ (Fig. $2 E, F$, black line) exhibited a small but significant bump (normal test; $p<0.01$ ) flanking the central peak, with a width corresponding to two to eight successive states that were significantly correlated. This bump was not observed when the original time series was scrambled (Fig. $2 E, F$, gray line). This phenomenon was observed and was significant in seven of seven free leeches and in seven of seven pinned leeches, with an average central peak width for $\rho$ series (Fig. $2 G$, black bars) of $5 \pm 2$ states for pinned leeches and $4 \pm 2$ states for free leeches. The same results were obtained for $\theta$ series (Fig. $2 G$, white bars), with a central width of $6 \pm 2$ and $3 \pm 1$ states, respectively, indicating the existence of a correlation between positions of successive stationary states. Values of $\rho$ and $\theta$ were not significantly crosscorrelated.

\section{Effects of sensory stimulation}

We further tested how the spatial distribution of leech locations changed as a consequence of localized sensory stimulation. For this purpose, we used seven pinned leeches (different leeches from those in Fig. 2). Five of them were monitored when one light was flickering, and the other two were monitored when two lights were flickering. Figure 3 shows some representative polar plots in which the angular distribution of the position of the leech during stationary states is reported. Without sensory stimulation, the distribution of stationary states for all tested leeches was isotropic 

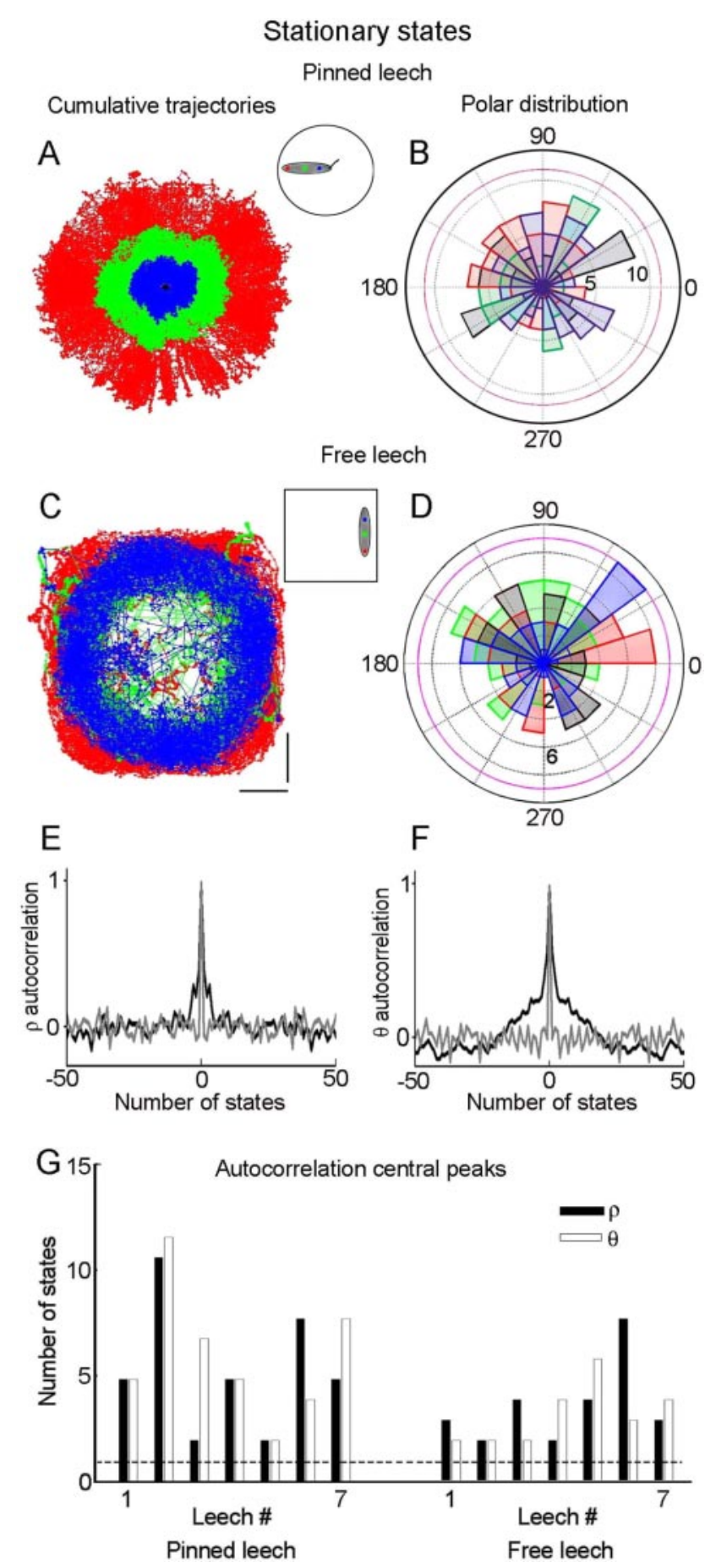

Figure 2. Analysis of stationary states. A, Displacement of a restrained leech (the position of the pin is marked with a black circle). The trajectories of the head, midbody, and tail, shown in red, green, and blue, respectively, show that the leech uniformly explores the surface of the dish. The inset shows a scheme of the restrained leech preparation. $\boldsymbol{B}$, Polar plot distribution of stationary states of four different restrained leeches (different color for each leech). The polar plot represents the number of stationary states for every direction, and the magenta circle shows that the distribution of stationary states does not have any statistically privileged direction ( $p>0.05)$. C, As in $\boldsymbol{B}$, but for a freely moving leech in a square dish. Scale bars, $3.5 \mathrm{~cm} . \boldsymbol{D}$, Polar plot distribution of stationary states of four freely moving leeches, which also demonstrates the spatial uniformity of exploration $(p>0.05) . \boldsymbol{E}, \boldsymbol{F}$, Superimposed plots of the autocorrelation of $\rho$ and $\theta$, respectively, for stationary states (black) and of the same series scrambled (gray). Black traces show a small but significant bump flanking the central peak, with a width corresponding to $5-10$ successive states. $\mathbf{G}$, Width of the autocorrelation central peaks for seven pinned (left) and seven free (right) leeches, representing the mean number of significantly correlated successive states for $\rho$ (black) and $\theta$ (white). The horizontal dotted line indicates the central spike expected even in the absence of any correlation.
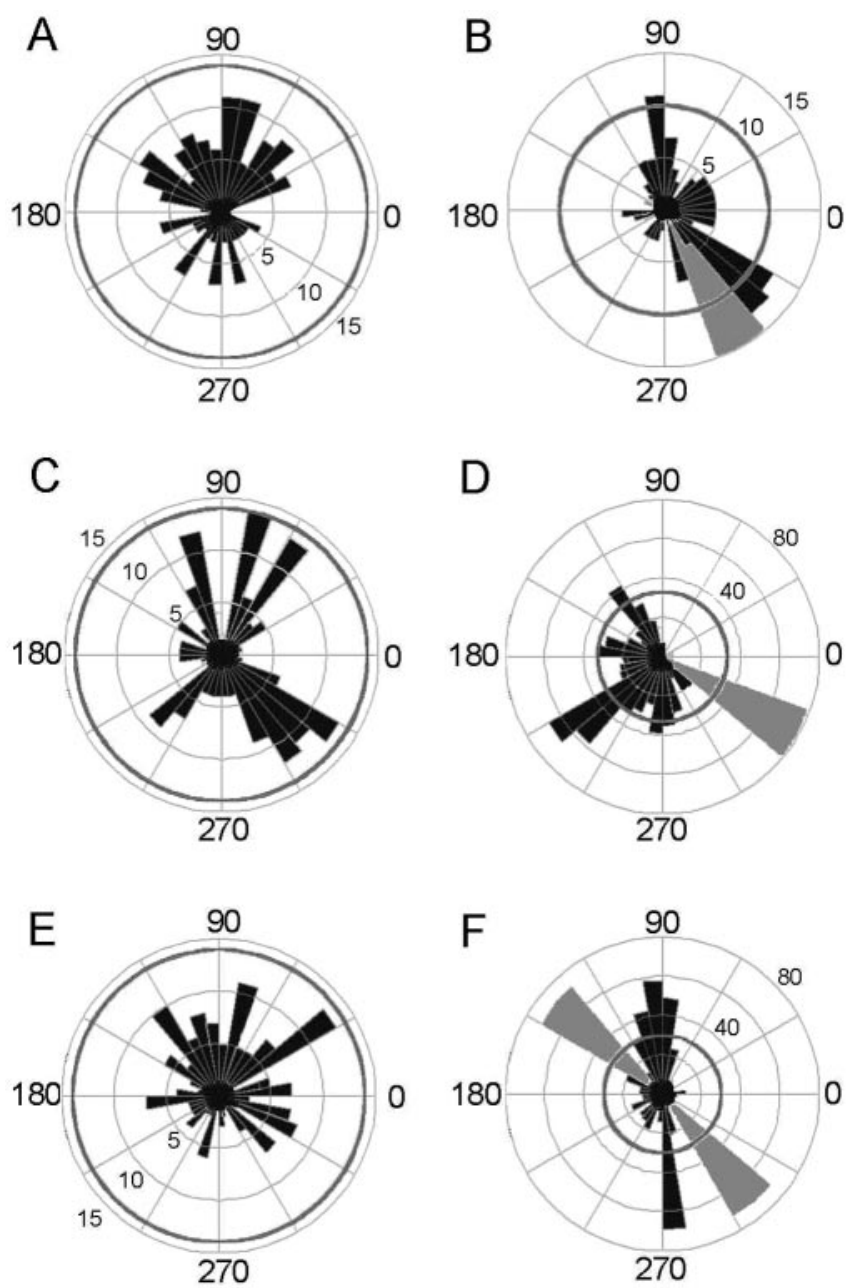

Figure 3. Effects of external stimulation. $A, C, E$, Polar plots with the distribution of the leech body orientations during stationary states in three different experiments using the pinned leech, showing that, in absence of any stimulation, the leech explores the dish uniformly with no preferential direction. $\boldsymbol{B}, \boldsymbol{D}, \boldsymbol{F}$, Polar plots of three different experiments using pinned leeches, showing the angular distribution of the position of the leech during stationary states in the presence of an external stimulation (light is represented as gray triangles). $\boldsymbol{F}$, Two (almost opposite) lights were used. Dark gray circles indicate 5\% confidence circle for the Poisson distribution of states per sector, as required by an isotropic behavior.

(the number of occurrences per sector followed a Poisson distribution; $p>0.05)$. As an example, the distribution of stationary states for three leeches is shown in Figure 3, $A, C$, and $E$, in which no sector exceeds the significance threshold (dark gray circle) for anisotropy; however, in four of five cases with one light and in two of two cases with two lights, the presence of the light broke this isotropy. Figure 3, $B, D$, and $F$, shows the angular distributions during stationary states for these same three leeches when the lights were switched on (the gray triangles indicates the orientation of the lights). In all three cases, some sectors were more populated than the statistical threshold for acceptance of isotropy $(p<0.05)$ (dark gray circle). Although a systematic relationship between orientation of light and preferred resting orientation of the leeches could not be found, in general the leeches avoided spending time under the light sources (Fig. 3D). Their preferred direction was often close to but not coincident with the light source, as if leeches moving toward the light source were constrained to proceed along a nearby direction (Fig. $3 B$ ). 


\section{Analysis of exploratory states}

Well resolved peaks characterized the smoothed power spectrum of swimming and crawling episodes, at 1.5 and $0.2 \mathrm{~Hz}$, respectively (Mazzoni et al., 2005). On the other hand, exploratory states were characterized by a $1 / f^{\S}$ distributed power spectrum (where $S$ is the slope of the power spectrum when plotted on a logarithmic scale), with no resolvable peaks. Furthermore, the shape of the smoothed power spectrum of $e(n)$ during exploratory episodes was very similar across different free and pinned leeches. Examples showing a superposition of four power spectra corresponding to different exploratory episodes observed in four pinned (reddish curves) and four free (bluish curves) leeches are reported in Figure 4, $A$ and $B$, respectively. Similarity among exploratory power spectra was better quantified by computing their $1 / f^{S}$ fit (Fig. $4 A, B$, black lines). The slope $S$ of the power spectrum was very similar across different pinned (red bars) and free (blue bars) leeches, as shown by the slope distributions reported in Figure $4 C$. The value of the slope $S$ is highly indicative of the kind of process analyzed and can be considered, in this case, as a measure of the correlation of the different movements composing the behavior (Berg, 1993; Szendro et al., 2001). The observed similarity suggests that, although very different and very irregular, all exploratory episodes belong to the same dynamics. For instance, the average slope of the power spectrum was $1.8 \pm 0.2$ for free (blue bars) and $2.0 \pm 0.2$ for pinned (red bars) leeches. Although small, the difference between average slopes in the two sets was significant (ANOVA test; $p<0.05$ ). The slope of the power spectrum is close to $1 / f^{2}$, indicating that exploratory movements approximately follow random walk dynamics (Berg, 1993).

Despite the quasi-periodic appearance of exploratory motion (Fig. 1 A, first light gray patch), its power spectrum was not associated with peaks at any frequency. Such a broadband spectrum is indicative of noise or nonlinear deterministic chaos. As a test for low-dimensional nonlinear deterministic chaos, which could be caused by, for example, the interactions of a few underlying motor oscillators, we examined whether exploratory time series could be predicted better by linear prediction, by nonlinear prediction, or by nonlinear prediction of the phase-randomized time series (Kantz and Schreiber, 1997); however, the best result was obtained using linear prediction (data not shown). Thus, we were not able to find evidence for simple low-dimensional chaos in exploratory time series.

We also verified that, during exploratory events, the leech visited the entire surrounding environment (Fig. $4 D$ ), with no preferential direction or location, and that the distribution of the head speed $V_{\text {head }}$ was Gaussian (Fig. $4 E$ ), as it was for the other behaviors (Mazzoni et al., 2005). Even if there was a characteristic speed for this behavior ( $\mu=35$ pixels per second) that was similar for pinned and free leeches, the distribution was broad $(\sigma=$ 13 pixels per second) compared with other behaviors (Mazzoni et al., 2005), as shown by the statistics for seven pinned and seven free leeches presented in Figure $4 F$. This broad distribution suggested heterogeneity of activated motor patterns, as expected for the highly irregular motion characterizing the exploratory behavior. Indeed, using the recurrence plot technique, it was possible to identify qualitatively various repeated, effectively deterministic motifs within the exploratory motion. Figure $5 A$ shows an example of a recurrence plot of a $1000 \mathrm{~s}$ section of exploratory behavior. A pattern of similar behavior repeated at two different points in the time series was observed as a diagonal line segment of slope -1 . All other similar patterns within the time series could be picked out as those diagonal line segments, which intersect the
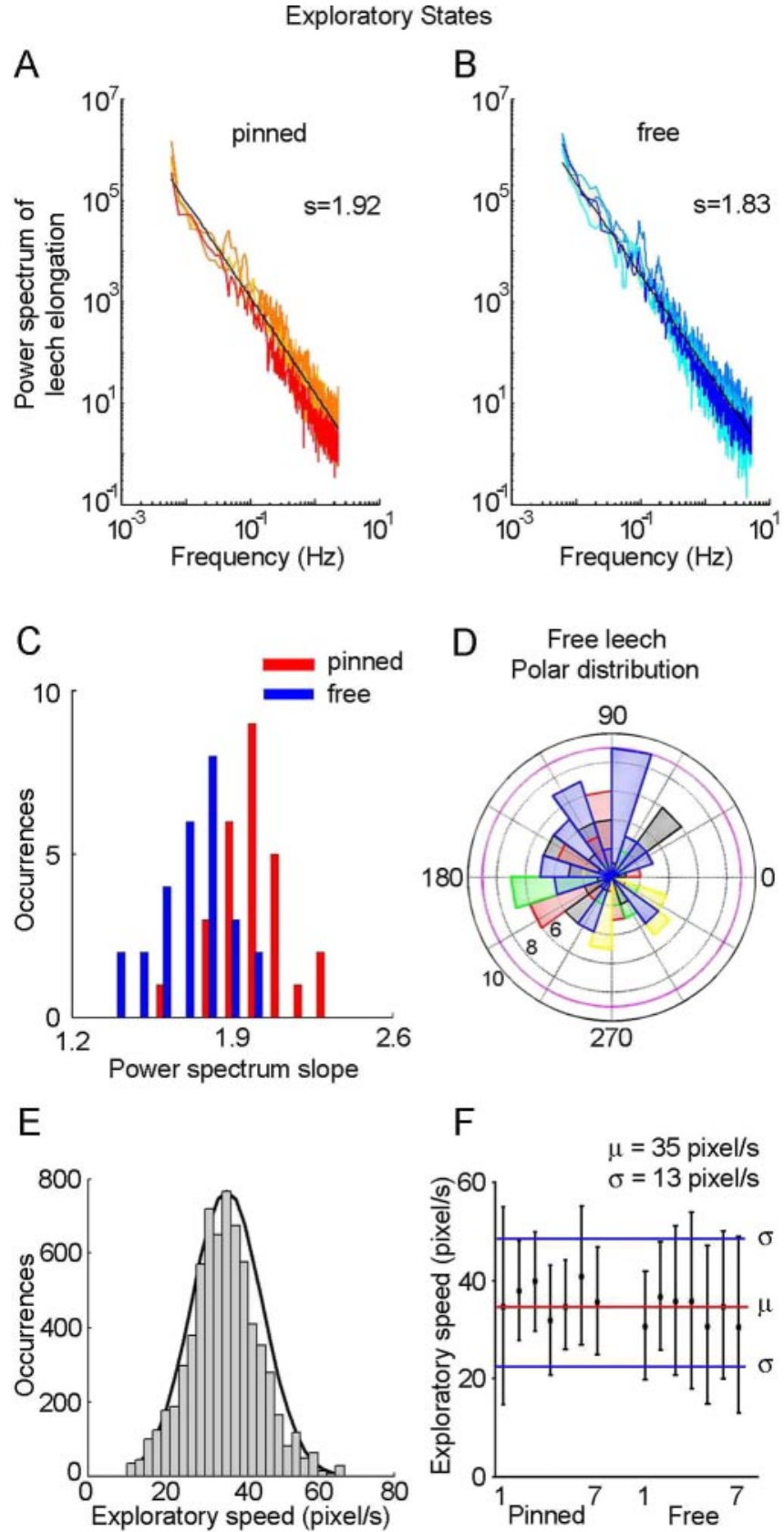

Figure 4. Spectral analysis for exploratory states. $\boldsymbol{A}$, Superposition of four smoothed power spectrums for $e(n)$ during four episodes (lasting 100 s each) of exploratory behavior observed in different leeches. The black line represents the $1 / f$ fit to one of the power spectra. The slope $S$ is indicated. $\boldsymbol{B}$, As in $\boldsymbol{A}$, but for four free leeches. $\boldsymbol{C}$, Distribution of the power spectrum slope $\boldsymbol{S}$ during exploratory episodes observed in 54 leeches, 27 pinned (in red) and 27 free (in blue). $\boldsymbol{D}$, Polar plot distribution of the position of the leech during exploratory states observed in five free leeches. Confidence interval is shown in magenta. $\boldsymbol{E}$, Maximum speed distribution during exploratory behavior (calculated for $10 \mathrm{~s}$ ) of one leech. This plot is well fit by a Gaussian distribution (black). $\boldsymbol{F}$, Mean and SD of the Gaussian fits for exploratory speeds of seven pinned leeches and seven free leeches.

same horizontal as the first identified diagonal. An example of this is shown in the inset; this pattern was found several times along the recurrent plot, and some of these are shown superimposed in Figure $5 C$ as pattern 4 . This pattern and some other recurrent patterns (Fig. $5 C$, patterns 1,11 ) are superimposed in black on a piece of the original time series in Figure $5 B$. These patterns show that the evolution of the exploratory behavior is similar at different times over periods lasting tens of seconds. 
We also performed the same analysis after Fourier transforming, randomizing the phase information, and taking the inverse transform. This phase-randomized surrogate series (Kantz and Schreiber, 1997) has the same power spectrum and autocorrelation as the original time series and therefore should give the same results if a linear stochastic process produced the data; however, there were in the original time series a number of recurrent patterns that were not observed in the phaserandomized surrogate. The 12 most common of these are shown in Figure 5C. They could be separated into several groups: (1) abrupt movements, as patterns 1 and 4, indicated in the original trace shown in Figure 5B, and (2) quasi-periodic fragments and oscillating extensions sandwiched by smooth oscillations, such as patterns 7-12.

\section{Transitions between behaviors}

A process in which the probability of transition between different states is determined only by the identity of the most recent state is said to be markovian. The exponential distribution of duration of exploratory, swimming, crawling, and stationary states (Fig. $1 D, G$ ) shows that every behavior or subcategory of behavior had a probability of ending per unit of time equal to $1 / \tau$ (where $\tau$ is its characteristic time constant). This is highly suggestive of a Markov process underlying the decision mechanisms that control transitions among different behaviors. To verify this possibility, transitions between different behavioral categories were analyzed. The probability $P(B / A)$ of the first-order (or one step) transition $A \rightarrow B$, i.e., the probability of state $k+1$ belonging to behavior $B$ if state $k$ belongs to $A$, was estimated for each pair of behaviors $(A, B)$, as described in Materials and Methods. The corresponding transition rate, i.e., the probability per unit of time for the leech of switching to behavior $B$ when it is in a state belonging to the behavior $A$, is then given by $P(B / A) / \tau_{A}$.

Figure $6 A$ shows, for a pinned leech, one-step transition probabilities between five different behaviors, for the first $(0-3 \mathrm{~h})$ and second $(3-6 \mathrm{~h}$ ) half of the experiment (black and white bars, respectively). In each panel, the transition probabilities in the first half of the recording session were not significantly different from those of the second half ( $\chi^{2}$ test for homogeneity of distributions; $p>0.05$ ), and the same result was obtained for four of five leeches examined. This indicates that the decision process was stationary in time.

Transition probabilities varied from leech to leech, but some properties of the Markov process were present in all leeches. For instance, peristalsis was always followed by stationary states (Fig. $6 A$, fourth panel), and transitions usually occurred among stationary, exploratory, and stationary states (Fig. 6A, first three panels). Some transitions, like swimming to crawling and crawling to swimming, were never observed in free leeches (six experiments); however, when the water level was not sufficient to fully cover the leech body, hybrid behaviors such as swimming-like oscillations superimposed on the elongation phase of crawling (Fig. 7) (Esch et al., 2002) and elongation followed by a swimming behavior were sometimes observed (data not shown). Fig- ure $7 A$ shows the time displacement of the $x$ and $y$ coordinates of the head (red), midbody (green), and tail (blue). Four crawling steps are shown during which the body was oscillating. In Figure $7 B$, the space displacement of the same hybrid behavior is shown. As can be seen, the leech was crawling from the middle to the bottom of the plot ( $y$ direction) with oscillatory movements $(x$ direction) superimposed on the elongation step. Note the difference between the $x$ and $y$ scales.

To verify that the sequence of behavioral states could be modeled as a first-order Markov process, we tested whether transitions between different states satisfied the Markov first-order dependence conditions, i.e., $P(B / A, X)=P(B / A)$, for every triplet $X \rightarrow A \rightarrow B$, where $P(B / A, X)$ is the probability of state $k+1$ belonging to behavior $B$ if the current state $k$ belongs to $A$ and the previous state $k-1$ belonged to $X$.

A $\chi^{2}$ test (see Materials and Methods) was used to test whether the set of two-step transition probabilities $P(B / A, X)$ was significantly different from the set of one-step transition probabilities $P(B / A)$. The $\chi^{2}$ statistics were computed taking into account all possible transitions between the three most frequent behaviors (stationary states, exploratory, and swimming) (Fig. 6A) in the five experiments that had an appreciable number of transitions $(n>5)$ expected for every triplet of states (for instance, $\mathrm{Sw} \rightarrow \operatorname{Exp}$ $\rightarrow$ Stat or Exp $\rightarrow$ Sw $\rightarrow$ Exp, in which Sw is swimming, Exp is exploratory, and Stat is stationary). The result of the test was that, for five of five experiments, the two-step probabilities were significantly different $(p<0.05)$ from the one-step probabilities, and therefore the sequences of identified behaviors could not be considered as first-order Markov processes; however, a comparison among the different terms in the $\chi^{2}$ statistics revealed that deviations from first-order dependence were attributable mainly to the transitions Exp $\rightarrow$ Stat $\rightarrow \operatorname{Exp}$ (Fig. 6B, first panel). This finding, together with the previous result that the distribution of the residence time for stationary states could be fit by a sum of two exponentials (Fig. $1 F$ ), strongly suggested that states identified as stationary were actually divided into two distinct subcat- 
Transition between states

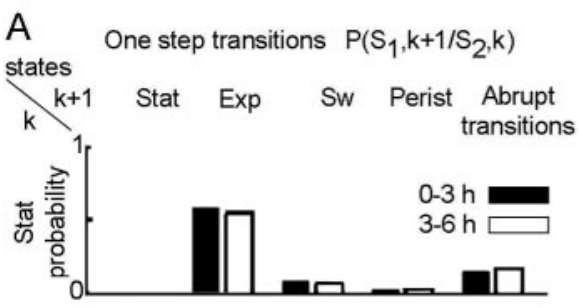

B
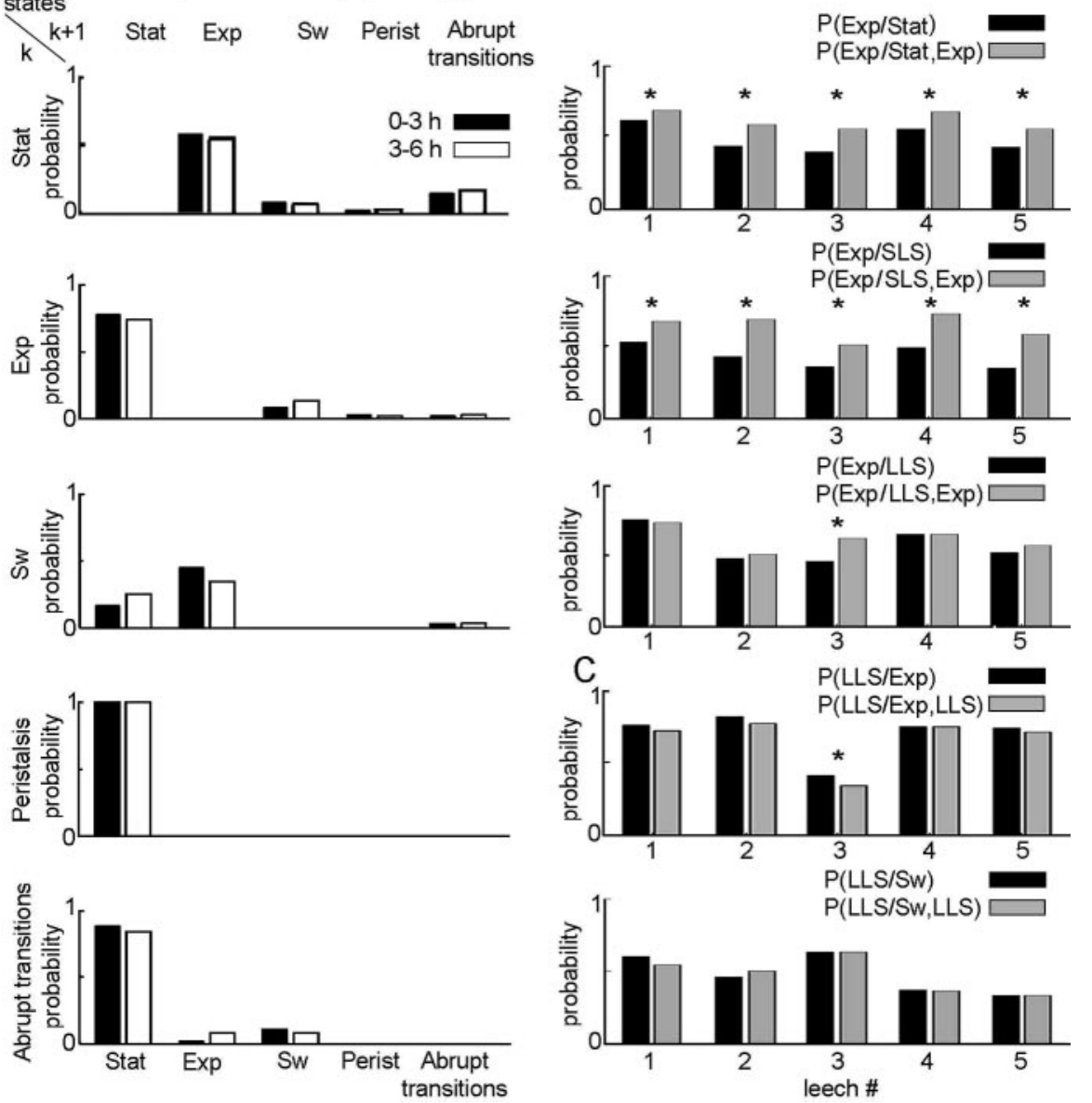

Figure 6. Analysis of transitions. A, Transition probabilities between different behaviors observed in one leech. Each row represents the transition probability between a starting state $(k$, on the left) and the following state $(k+1$, on top). Transition probabilities have been calculated for the first half (black) and second half of the experiment (white). Data were collected from a pinned leech preparation lasting $12 \mathrm{~h}$. $\boldsymbol{B}$, The first panel shows probability of transition for state $k+1$ being exploratory if state $k$ was stationary (black) and probability of the same transition conditional on state $k-1$ being exploratory (gray). This second two-step probability was always higher, and the overall transition rates were significantly different $\left({ }^{*} p<0.05\right)$ from the one-step conditional probabilities. The second and third panels show the same analysis but for SLS states (middle) and LLS states (bottom). For SLS states, the conditional probability was always higher, and the overall transition rates were again significantly different $\left({ }^{*} p<0.05\right)$ from the one-step conditional probabilities. For LLS states, however, the one- and two-step conditional probabilities were not significantly different for four of five experiments $\left({ }^{*} p<0.05\right)$. C, Top, Two-step transitions to LLS states from exploratory states (black) and from exploratory states after an LLS state (gray). The absence of significant difference ( $\chi^{2}$ test for homogeneity of distributions; ${ }^{*} p<0.05$ ) between the one- and two-step conditional probabilities shows that transition probabilities do not depend on the previous behavioral history. Bottom, The same comparison is done for swimming transition probabilities, obtaining the same result $\left({ }^{*} p<0.05\right)$. Stat, Stationary; Exp, exploratory; Sw, swimming.

egories. Therefore, based on the residence time distribution, we divided stationary states into two subcategories: short-lived stationary (SLS) states (duration $<10 \mathrm{~s}$ ) (Fig. $1 \mathrm{~F}$, first data point) and LLS states (duration $>10 \mathrm{~s}$ ).

The $\chi^{2}$ test was then repeated to include those transitions involving only SLS states or those involving LLS states. When only short stationary states were included, the two-step probabilities were still significantly different $\left(\chi^{2}\right.$ test; $p<0.05$; five of five experiments) from one-step probabilities. This again was because $P($ Exp/SLS,Exp) was always higher than $P($ Exp/SLS) (Fig. $6 B$, second panel). On the other hand, when only long stationary states were included in the statistics, conditioned one-step and two-step probabilities were not significantly different in four of five experiments $\left(\chi^{2}\right.$ test; $\left.p>0.05\right)$. A comparison between
$P($ Exp/LLS,Exp) and $P(\operatorname{Exp} /$ LLS $)$ is shown in Figure $6 B$ (third panel).

Overall, this analysis suggested that LLS and SLS states had very different properties. Long stationary states behaved like exploratory and swimming behaviors, and the transition probabilities were not affected by the previous behavior. On the contrary, during a short stationary state, the leech remembered the previous behavior and had the tendency to repeat it. This happened mainly when the leech was exploring. In fact, many of the short pauses were between two exploratory states, and most of them adjoin at least one exploratory state (32 and 74\%, respectively, of SLS states for nine leeches in which both SLS and exploratory states had an appreciable number of occurrences). This suggests that SLS states are pauses that do not have to be considered as independent from the exploratory states. Therefore, we repeated the statistical test for first-order Markov dependence by absorbing each SLS state that bordered an exploratory state into the exploratory state itself. Such paired states contributed, on average, 15\% of the total number of stationary states and $24 \%$ of the number of exploratory states (nine leeches). Figure 6C shows some of the resulting two-step and one-step transition probabilities after this adjustment in the classification of behaviors. Here, transitions from exploratory (Fig. 6C, top) and from swimming (bottom) to LLS states are compared, when the state preceding the swimming or exploratory state is either arbitrary (black) or LLS (gray). For four of five experiments, two-step probabilities were not significantly different $(p>0.05)$ from the one-step probability set, indicating that the underlying transition dynamics behaves, in essence, as a first-order Markov process.

\section{Discussion}

The aim of the present work was to establish a basis for the quantitative understanding of leech behavior and decision processes. The proposed automatic classification is able to recognize and classify most of the motions; only a small fraction, $\sim 10 \%$, of leech motion remains unclassified. This percentage appears to be a combination of different behaviors, drifts, and oscillations of body parts. In addition to the well characterized behaviors such as swimming and crawling, it was possible to identify and study a more complex category of movements, the exploratory behavior, which has never been investigated quantitatively before.

\section{Flow of leech behavioral decisions as a first-order Markov process}

The results shown in this study (Figs. 1, 6) suggest that the time structure of leech behavior is well described as a first-order 
Markov process with transitions among various different behavioral categories, as illustrated in Figure $8 \mathrm{~A}$. Transition probabilities among different behavioral categories are likely to depend on the spontaneous neural activity of the animal brain as well on proprioceptive and sensory information. The discovery of many command interneurons in the subesophageal ganglion of the leech, the locations of which are indicated in the diagram in Figure $8 B$, offers a neurobiological basis for the scheme of Figure $8 \mathrm{~A}$.

Neurons Tr1, SE1, and R3b1 initiate swimming (Brodfuehrer and Friesen, 1986a,b,c; Brodfuehrer et al., 1995; Thorogood and Brodfuehrer 1995; Shaw and Kristan, 1997; Esch et al., 2002), whereas neurons SIN1 and Tr2 terminate swimming (Brodfuehrer and Burns, 1995; O'Gara and Friesen, 1995). The firing of neuron R3b1 (Esch et al., 2002) elicits both swimming and crawling or a hybrid behavior (similar to that shown in Fig. 7), depending on the sensory environment (the depth of the fluid surrounding the leech); however, leech behavioral decisions are likely to depend on a much more distributed neural dynamics than just the activity of this set of neurons. In fact, behavioral choice experiments have shown that most of these neurons are multifunctional, i.e., they are activated during different behaviors such as swimming and whole-body shortening ( $\operatorname{Tr} 1$ and SE1) (Shaw and Kristan, 1997) or swimming and crawling (R3b1) (Esch et al., 2002). Moreover, recordings of the neural activity descending from the head ganglion in the connective have shown a massive coactivation of many unidentified interneurons during swimming episodes (Brodfuehrer and Burns, 1995). Finally, direct stimulation of identified command neurons produces highly variable behavioral responses and often does not elicit any kind of behavior (Brodfuehrer and Friesen, 1986b). The same variability is encountered when strong mechanosensory stimuli are used to elicit swimming (Grobstein, 1994; Brodfuehrer and Burns, 1995). As a consequence, the resulting stimulus-response curves do not have a clear stimulus level threshold for reproducible initiation of swimming. Overall, these findings suggest that leech behavioral decisions are controlled by the dynamics of a distributed neural network that defines the internal state of the leech CNS. This dynamics, because of its distributed nature and the intrinsic variability of leech interneuron and motoneuron firing (Zoccolan et al., 2002), can be regarded as a temporally structured probabilistic process. Therefore, we believe that the approach presented here (i.e., modeling leech behavioral data as a first-order Markov process) is at present the most effective for quantifying and characterizing the complexity of decision making in the leech CNS.

A future challenge in the study of leech behavioral decisions will be to compare transition probabilities in leeches moving in an unstructured sensory environment (as done in this work) and in leeches exposed to specific sensory stimuli or during selective intracellular stimulation of command neurons (or extracellular stimulation of the connective fibers). Another challenge will be to correlate the spontaneous activity of the identified command neurons with the overall Markov process controlling the transitions among different behaviors. The biophysical properties of these neurons and of their synapses are likely to take part in establishing the duration of different behaviors and transitions from one behavior to another. Because the duration distribution of long stationary states, swimming, and crawling episodes is on the order of tens of seconds, the dynamics of activation and deactivation of these command neurons should occur on a time scale much longer than the usual synaptic delays. Unraveling the biophysical and neurobiological mechanisms underlying such long-term neuronal modulations appears to be the key to understanding the process of behavioral decision making in the leech.

\section{Spatial distribution during stationary states}

Although the transitions among different behavioral states satisfied the Markov property of "no memory," a significant correlation between the orientations (as well as the elongations) of the leech body in consecutive stationary states has been found (Fig. $2 E-G)$. This may be partially accounted for by a sort of "biomechanical memory" or inertia of the leech body that, in between two close stationary states, does not have time to change its location fully; however, because the correlation has been estimated by taking into account all identified stationary states, the existence of neural circuits that effectively implement a short-term spatial memory cannot be excluded. This possibility is also supported by the fact that, in the absence of directional sensory stimuli, the distribution of leech body orientations during stationary states is isotropic (Fig. $2 A-D$ ). Therefore, the correlation between consecutive stationary orientations cannot be attributed to the tendency of the leech to rest along some preferred direction(s). On the other hand, the finding that bright flashing lights located at specific orientations can destroy the isotropy of the leech resting orientations (Fig. 3) is easily explained as the direct and continuous effect of the sensory stimuli on the decision process, without the need to hypothesize any short-term spatial memory.

\section{Exploratory behavior}

The exploratory behavior is a highly irregular, apparently unpredictable motion, however, in which we were able to identify various recurring patterns or motifs, suggestive of a deterministic dynamics (Fig. 5). Although a test for a deterministic dynamic attractor failed when applied to a long piece (1000 s) of exploratory time series, these recurrent motifs were consistently found only in the original time series but not in its phase-randomized surrogate. This suggests that nonlinear neural pattern generators could actually produce deterministic pieces of exploratory motion embedded in an overall effectively random explorative behavior. Also, the exploratory motion could simply be continuously broken by the sensory feedback provided by the external environment (which is likely to be maximal during exploration), 


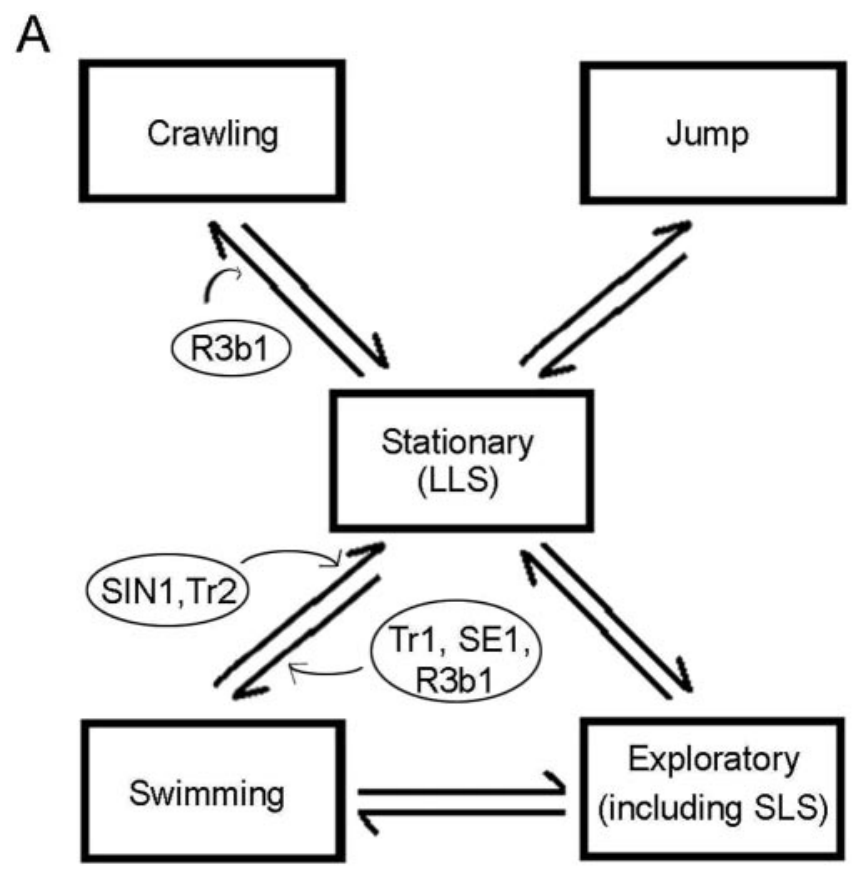

B

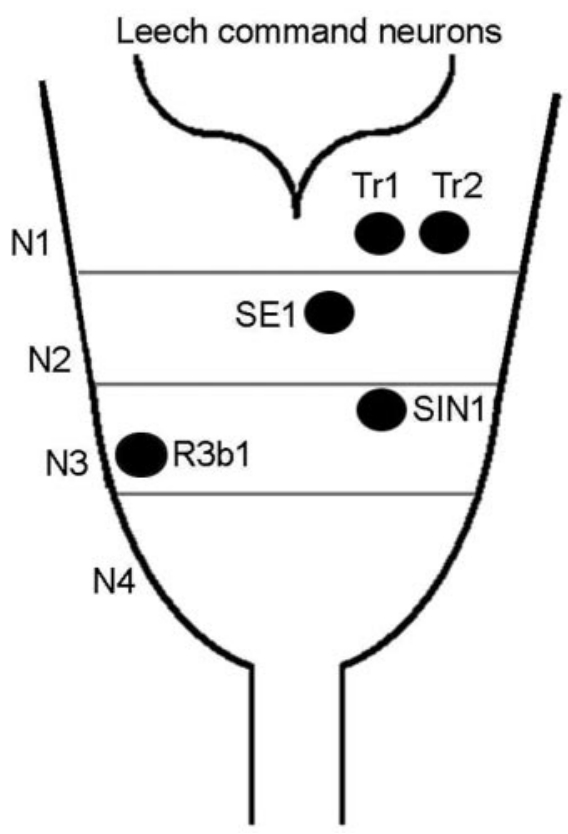

Figure 8. Behavior of the leech and transitions among behavioral categories. A, Five behaviors and the transitions between them are shown. Stationary states (LLS) are those with a duration of $\geq 10 \mathrm{~s}$. The exploratory states included the SLS, with a duration of $<10 \mathrm{~s}$. The transitions among behavioral categories are probably controlled by the firing of the command neurons. The known command neurons and their location in the head subesophageal ganglia are shown in $\boldsymbol{B}$. N1-N4 represent the four neuromeres of the head ganglion.

thereby preventing the existence of a global dynamic attractor. In any case, the existence of such motifs is likely to have a deep physiological and behavioral relevance.

Despite the heterogeneity of the activated motor patterns, as suggested by the broad distribution of the head velocity (Fig. $4 E, F$ ) and by the variety of identified recurrent motifs (Fig. 5), the residence time distribution of exploratory states could be fit by a single exponential (Fig. $1 D$ ). Moreover, transition probabil- ities involving exploratory states satisfied the Markov first-order dependence conditions as well as stationary and swimming states (Fig. 6C). This strongly suggests that the decision process underlying initiation and termination of the exploratory behavior should share the same neural architecture that triggers or inhibits more stereotyped behaviors such as swimming or crawling. Therefore, we are tempted to speculate that a network of "exploratory" command neurons should exist in the leech head ganglion. Similar to what was found for swimming, this high-level network could simply decide when to start and stop an exploratory episode, whereas more specialized intra-ganglia and inter-ganglia circuits could work as nonlinear pattern generators of the exploratory patterns. These speculations will be the subject of future investigations.

Short pauses that adjoined exploratory states were included in the exploratory states themselves, because their statistics were clearly distinct from that of longer stationary states (Figs. $1 F, 6 B$ ). Therefore, short pauses could be considered a special kind of exploratory pattern. They could represent "intermittent locomotion," in which an animal uses brief pauses to improve the quality of sensory information to guide its behavior in response to external stimuli. This behavior has been observed in animals ranging from protozoans to mammals (Kramer and McLaughlin, 2001).

\section{Final remarks}

This work shows that the spontaneous behavior of the leech can be quantitatively analyzed and characterized for long periods of time, providing enough data for an adequate statistical analysis. Formal schemes for the behavior can then be compared with experimental data, and mathematical models can be obtained. Such an approach has already been applied successfully to the analysis of behavior of different species, from invertebrates to primates (Bressers et al., 1995; Haccou and Meelis, 1992; Chen et al., 2002); however, the leech offers the great advantage of accessibility of the nervous system to intracellular and extracellular recordings and a growing knowledge of the command interneurons responsible for shaping its behavior. Revealing the neural correlates of the Markov process and of the exploratory patterns found in the present investigation could lead to a better general quantitative theory of animal behavior relating to neuroeconomics (Glimcher, 2003) and game theory (Schultz, 2004).

\section{References}

Baader AP, Kristan Jr WB (1992) Monitoring neuronal activity during discrete behaviors: a crawling, swimming and shortening device for tethered leeches. J Neurosci Methods 43:215-223.

Barraclough DJ, Conroy ML, Lee D (2004) Prefrontal cortex and decision making in a mixed-strategy game. Nat Neurosci 7:404-410.

Bennett MV (1997) Gap junctions as electrical synapses. J Neurocytol 26:349-366.

Bennett MV (2000) Electrical synapses, a personal perspective (or history). Brain Res Brain Res Rev 32:16-28.

Berg HC (1993) Random walks in biology. Princeton: Princeton UP.

Bressers WM, Kruk MR, Van Erp AM, Willekens-Bramer DC, Haccou P, Meelis E (1995) A time-structured analysis of hypothalamically induced increases in self-grooming and activity in the rat. Behav Neurosci 109:1158-1171.

Britten KH, Van Wezel RJ (1998) Electrical microstimulation of cortical area MST biases heading perception in monkeys. Nat Neurosci 1:59-63.

Brodfuehrer PD, Burns A (1995) Neuronal factors influencing the decision to swim in the medicinal leech. Neurobiol Learn Mem 63:192-199.

Brodfuehrer PD, Friesen WO (1986a) Initiation of swimming activity by trigger neurons in the leech subesophageal ganglion. I. Output connections of Tr1 and Tr2. J Comp Physiol [A] 159:489-502.

Brodfuehrer PD, Friesen WO (1986b) Initiation of swimming activity by 
trigger neurons in the leech subesophageal ganglion. II. Role of segmental swim-initiating interneurons. J Comp Physiol [A] 159:503-510.

Brodfuehrer PD, Friesen WO (1986c) Initiation of swimming activity by trigger neurons in the leech subesophageal ganglion. III. Sensory inputs to Tr1 and Tr2. J Comp Physiol [A] 159:511-519.

Brodfuehrer PD, Thorogood MS (2001) Identified neurons and leech swimming behavior. Prog Neurobiol 63:371-381.

Brodfuehrer PD, Parker HJ, Burns A, Berg M (1995) Regulation of the segmental swim-generating system by a pair of identified interneurons in the leech head ganglion. J Neurophysiol 73:983-992.

Chen S, Lee AY, Bowens NM, Huber R, Kravitz EA (2002) Fighting fruit flies: a model system for the study of aggression. Proc Natl Acad Sci USA 99:5664-5668.

Ditterich J, Mazurek ME, Shadlen MN (2003) Microstimulation of visual cortex affects the speed of perceptual decisions. Nat Neurosci 6:891-898.

Drewes CD (1984) Escape reflexes in earthworms and other annelids. In: Neural mechanisms of the startle response (Eaton RC, ed), pp 43-91. New York: Plenum.

Eckmann JP, Oliffson-Kamphorst S, Ruelle D (1987) Recurrence plots of dynamical systems. Europhys Lett 4:973-977.

Esch T, Mesce KA, Kristan WB (2002) Evidence for sequential decision making in the medicinal leech. J Neurosci 22:11045-11054.

Freedman DJ, Riesenhuber M, Poggio T, Miller EK (2001) Categorical representation of visual stimuli in the primate prefrontal cortex. Science 291:312-316.

Furshpan EJ, Potter DD (1959) Transmission at the giant motor synapses of the crayfish. J Physiol (Lond) 145:289-325.

Glimcher P (2002) Decisions, decisions, decisions: choosing a biological science of choice. Neuron 36:323-332.

Glimcher P (2003) Decisions, uncertainty, and the brain: the science of neuroeconomics. Cambridge, MA: MIT.

Gold JI, Shadlen MN (2002) Banburismus and the brain: decoding the relationship between sensory stimuli, decisions, and reward. Neuron 36:299-308.

Green DM, Swets JA (1966) Signal detection theory and psychophysics. New York: Wiley.

Grobstein P (1994) Variability in brain function and behavior. In: Encyclopedia of human behavior (Ramachandran VS, ed), pp 447-458. New York: Academic.
Haccou P, Meelis E (1992) Statistical analysis of behavioural data: an approach based on time-structured models. Oxford: Oxford UP.

Kantz H, Schreiber T (1997) Nonlinear time series analysis. Cambridge, UK: Cambridge UP.

Kramer DL, McLaughlin RL (2001) The behavioral ecology of intermittent locomotion. Am Zool 41:137-153.

Mazzoni A, Garcia-Perez E, Zoccolan D, Graziosi S, Torre V (2005) Quantitative characterization and classification of leech behavior. J Neurophysiol 93:580-593.

Montague PR, Berns GS (2002) Neural economics and the biological substrates of valuation. Neuron 36:265-284.

O'Gara BA, Friesen WO (1995) Termination of leech swimming activity by a previously identified swim trigger neuron. J Comp Physiol [A] 177:627-636.

Priestley MB (1981) Spectral analysis and time series. London: Academic.

Salzman CD, Britten KH, Newsome WT (1990) Cortical microstimulation influences perceptual judgments of motion direction. Nature 346:174-177.

Schnizler M, Clauss W (1998) Characterization of a voltage-dependent conductance in the basolateral membrane of leech skin epithelium. J Comp Physiol [B] 168:295-302.

Schnizler M, Buss M, Clauss W (2002) Effects of extracellular purines on ion transport across the integument of Hirudo medicinalis. J Exp Biol 205:2705-2713.

Schultz W (2004) Neural coding of basic reward terms of animal learning theory, game theory, microeconomics and behavioural ecology. Curr Opin Neurobiol 14:139-147.

Shaw BK, Kristan Jr WB (1997) The neuronal basis of the behavioral choice between swimming and shortening in the leech: control is not selectively exercised at higher circuit levels. J Neurosci 17:786-795.

Szendro P, Vincze G, Szasz G (2001) Pink-noise behavior of biosystems. Eur Biophys J 30:227-231.

Thorogood MS, Brodfuehrer PD (1995) The role of glutamate in swim initiation in the medicinal leech. Invert Neurosci 1:223-233.

Tinbergen N (1951) The study of instinct. Oxford: Clarendon.

Zoccolan D, Pinato G, Torre V (2002) Highly variable spike trains underlie reproducible sensorimotor responses in the medicinal leech. J Neurosci 15:10790-10800.

Zucker RS (1972) Crayfish escape behavior and central synapses. I. Neural circuit exciting lateral giant fiber. J Neurophysiol 35:599-620. 